\title{
SYMPOSIUM ON RHEUMATOID ARTHRITIS
}

\author{
STоскноLм, 1957
}

A symposium on the differential diagnosis and treatment of rheumatoid arthritis was held at the Karolinska Sjukhuset, Stockholm, Sweden, on April 15-18, 1957. Abstracts of 25 papers contributed by speakers from fourteen countries, and the titles of five papers for which abstracts are not available, are given below.

(1) Is there a Pathological Definition of Rheumatoid Arthritis? By D. H. Collins (Professor of Pathology, University of Sheffield, England).

Rheumatoid arthritis is a disease, the cause of which is still unknown, that is characterized by chronic inflammation of one or more joints. The principal lesion is a chronic synovitis with proliferation of the stratum synoviale and massive lymphoid or plasma-cell infiltrations often forming foci. This may be seen in tendon sheaths as well as in joints. Tubercle follicles, abscesses, and bacteria are absent. Synovitis is succeeded by the erosion of cartilage and bone by granulation tissue, and, unless arrested by treatment or remission, there is progressive disorganization of the joint by the granulomatous process.

Non-articular lesions are common, though not essential, features of the disease. They take the form of:

(1) subcutaneous nodules;

(2) nodules in the fibrous structures of viscera;

(3) microscopical foci of the lymphoid cells in somatic tissues;

(4) other inflammatory lesions, such as arteritis, pericarditis, and dermatitis.

All lesions of rheumatoid arthritis are granulomatous, either diffuse, as in the joints, or focal, as in the nodules. The granulomatous reaction is composed of:

(a) a proliferation of vascular and connective tissue cells;

(b) accumulation of lymphocytes, plasma cells, and less_numerous granulocytes;

(c) necrosis of connective tissue;

(d) exudate, often rich in mucopolysaccharides, that with $(c)$ gives rise to the picture of "fibrinoid degeneration".

There is no single histopathological change that will identify rheumatoid arthritis, but, as is the case in most diseases, the broader the view the more distinctive the pathological picture becomes.
(2) Histological Observations on a Tonsil in a Case of Rheumatoid Arthritis. By G. DomaGK (WuppertalElberfeld, Germany).

In a case of rheumatoid arthritis removal of the tonsils was apparently followed by cure of the arthritis. Histological examination of the excised tonsils showed enormous amounts of bacteria. Thus, primary chronic polyarthritis may be due to an infection, and may be cured by tonsillectomy and intensive chemotherapy with small doses of sulphonamide and antibiotics before and after the operation.

(3) Synovial Specimens obtained by Knee Joint Biopsy. By William M. Mikkelsen, Ivan F. Duff, Willliam Castor, and A. James French (Ann Arbor, Michigan), and Henry A. Zevely (San Luis Obispo, California).

Major complications have not occurred on the 160 occasions that synovial needle biopsy (Polley-Bickel, 1951) has been performed in 142 selected patients. This convenient technique may be developed into an invaluable teaching aid in rheumatic diseases and is a ready source of material for histochemical study and tissue culture. Failure to obtain adequate specimens occurred in less than 4 per cent. of cases. Synovial morphological characteristics must always be correlated with pertinent clinical and laboratory information. Histological abnormalities proved to be of definite diagnostic aid in sixty cases; in the remainder, the changes were non-specific or too slight to suggest a definite diagnosis. Urate deposits in six of fifteen cases of gout constituted the only observed pathognomonic change; otherwise, biopsy was most often fruitful in rheumatoid arthritis, neuropathic arthropathy, and specific infectious arthritis. No essential difference in synovial appearance was observed between rheumatoid arthritis and juvenile rheumatoid arthritis, peripheral joint involvement in ankylosing spondylitis, "rheumatoid" arthritis with psoriasis, and Sjögren's syndrome with rheumatoid arthritis.

(4) Do the Common Features exceed the Distinguishing Features in Rheumatoid Arthritis and Ankylosing Spondylitis? By V. FORBECH (Oslo, Norway).

Ankylosing spondylitis seems to be a rather common disease in Norway and its frequency in females seems to be greater than is generally stated.

Judging from the patients treated in the Hospital for Rheumatic Diseases in Oslo (Oslo Sanitetsforenings 
Sykehus, 140 beds), to which people from all over Norway are admitted, the ratio of rheumatoid arthritis to ankylosing spondylitis is about $14: 1$ in women and about $1 \cdot 4: 1$ in men. The sex ratio is about 3 to 4 women to one man in rheumatoid arthritis, and about 3 to 4 men to one woman in ankylosing spondylitis.

The yearly average, including admissions for rheumatoid arthritis and ankylosing spondylitis, for the 10 years 1947-1956, which form about two-thirds of all admissions, are shown in the following Table:

\begin{tabular}{|c|c|c|c|c|c|c|}
\hline \multirow{2}{*}{ Patients } & \multicolumn{2}{|c|}{ Women } & \multicolumn{2}{|c|}{ Men } & \multicolumn{2}{|c|}{ Total } \\
\hline & No. & $\begin{array}{l}\text { Per } \\
\text { cent. }\end{array}$ & No. & $\begin{array}{c}\text { Per } \\
\text { cent. }\end{array}$ & No. & $\begin{array}{l}\text { Per } \\
\text { cent. }\end{array}$ \\
\hline $\begin{array}{l}\text { Rheumatoid Arthritis .. } \\
\text { Ankylosing Spondylitis }\end{array}$ & $\begin{array}{r}392 \\
29\end{array}$ & $\begin{array}{r}62 \cdot 5 \\
4 \cdot 6\end{array}$ & $\begin{array}{r}119 \\
87\end{array}$ & 19 & $\begin{array}{l}511 \\
116\end{array}$ & $\begin{array}{l}81 \cdot 5 \\
18 \cdot 5\end{array}$ \\
\hline Total & 421 & $67 \cdot 1$ & 206 & $32 \cdot 9$ & 627 & 100 \\
\hline
\end{tabular}

Several factors suggest that ankylosing spondylitis is a disease entity clearly separated from rheumatoid arthritis:

The lack of ordinary subcutaneous rheumatic nodules.

The lack of the haemagglutinating serum factor in the Waaler-Rose test.

The frequency of the typical, acute, recurrent, benign iritis in ankylosing spondylitis (about 25 per cent. in our first series of 485 cases, 393 men and 92 women).

The different behaviour of the spine; even the most crippled rheumatoid arthritic usually retains a full range of movement in the lumbar and thoracic spine.

Further investigations to find still more factors separating rheumatoid arthritis and ankylosing spondylitis may assist in discovering the aetiology of the rheumatic diseases.

(5) Open Questions in Rheumatoid Arthritis. By Svend Clemmesen (Copenhagen, Denmark).

Important precipitating factors must exist in rheumatoid arthritis, when the fact is taken into consideration that in a series of pairs of identical twins, both twins acquired the disease in only 45 per cent. of cases. In rheumatoid spondylitis, on the other hand, the importance of hereditary factors is evident in twins.

In view of the action of gold salts in rheumatoid arthritis, gold metabolism and the influence of gold on reticulo-endothelial tissue should be studied.

(6) The Borderline between Collagenosis and Rheumatism. By E. MARTIN (Geneva, Switzerland).

The limits of rheumatism are not defined and there is a no-man's-land between rheumatoid arthritis and the collagen diseases. The nature and aetiology of the collagen diseases may be determined by studying this borderland, as for example, in cases of scleroderma associated with rheumatoid arthritis or with ankylosing spondylitis, or in cases of rheumatoid arthritis with positive L.E. cells. An indeterminate type of collagenosis is found too frequently with cancer of the digestive tract for the association to have arisen by chance. Rheumatoid arthritis must be studied as a systemic disease and not merely as an inflammatory joint condition.

(7) Clinical Investigation of the L.E. Cell Phenomenon in Patients with Rheumatoid Arthritis. By J. GosLings and J. H. KIEviTs (Leiden, Holland).

A positive L.E. cell phenomenon was found in 17 per cent. of a series of patients with rheumatoid arthritis (Kievits, Goslings, Schuit, and Hijmans, 1956).

In comparing the patients with L.E. cells with those without significant differences were found in the frequency of occurrence of eight signs and symptoms in the two groups.

Rheumatoid arthritis syndrome with positive L.E. cell phenomenon was distinguished from systemic lupus erythematosus by a quantitative evaluation of the phenomenon, the number of L.E. cells being on the whole significantly lower in rheumatoid arthritis than in systemic lupus erythematosus $(P<0 \cdot 01)$.

In comparing the direct method of Zimmer and Hargraves with our indirect method, it appears that the decrease in the number of L.E. cells per 1,000 granulocytes from the direct to the indirect method is less in systemic lupus than in rheumatoid arthritis (Kievits and Schuit, 1957).

(8) Does Systemic Lupus Erythematosus frequently simulate Rheumatoid Arthritis? By F. LeNOCH (Prague, Czechoslovakia).

Systemic lupus erythematosus seems to occur in Central Europe less frequently than in Western Europe. This fact could be explained by the less abundant use of antibiotics and chemotherapeutic agents than elsewhere. To the six questions asked by Rogoff and others (1956) at Aix-les-Bains, the authors add a further one:

Have all the L.E. cells considered as such hitherto really been L.E. cells, or would some of them now be classified as A cells or B cells according to Heller and Zimmerman?

In 150 patients suffering from rheumatoid arthritis, the authors found definite L.E. cells in only 2 per cent. (one male and two females), and A and B cells in 23 patients.

Even if one of the three actually had systemic lupus erythematosus, the two others were certainly suffering from rheumatoid arthritis.

If all 26 patients in whom L.E. cells and the similar $A$ and B cells are counted, we obtain a percentage of $17 \cdot 3$ (similar to the number reported by Goslings and his co-workers, although they did not take the rosettes as a positive finding).

The series, like that of Gosling's, was non-selected, so that the numbers were lower than those of Freyberg, who chose only atypical cases or those with complications.

Typical L.E. cells were found only in 2 per cent. of the 150 patients with rheumatoid arthritis.

Therefore the presence of L.E. cells, otherwise specific 
for systemic lupus erythematosus, do not refute a diagnosis of rheumatoid arthritis.

(9) Reversal of the Urinary Excretion Ratio of Nor-adrenaline : Adrenaline in Rheumatoid Arthritis. By L. J. Michotte (Brussels, Belgium).

The synergy of action of nor-adrenaline and adrenaline with hydrocortisone, the metabolism of which is not disturbed in rheumatoid arthritis, and the significant appearance of rheumatic states, identical to rheumatoid arthritis, during prolonged treatment with hydralazine justify a study of the sympathetic chemical transmitter, nor-adrenaline.

The 24-hr urinary excretion of nor-adrenaline and adrenaline gives an exact idea of the metabolism of these substances. This urinary excretion varies quantitatively according to the physical activity of the subject, but the excretion ratio Nor-adrenaline : Adrenaline is constant, according to the methods used as $5: 3 \cdot 5$.

In fifteen patients with untreated rheumatoid arthritis the mean ratio was $1: 4$; the excretion of nor-adrenaline in six cases being nil.

The decrease or suppression of the urinary excretion of nor-adrenaline may be explained either by a renal disorder that hinders the excretion by an insufficiency of secretion, or by an increased destruction.

(a) The injection of 1,000 gammas of nor-adrenaline, producing a renal overloading, brings the noradrenalinaemia from an average of 0.95 gammas per $12 \mathrm{hrs}$, to an average of 5.38 gammas per $12 \mathrm{hrs}$, and the ratio of excretion from 0.5 to $1 \cdot 9$, which shows that the renal excretion potential of nor-adrenaline is unchanged.

(b) The ingestion of $60 \mathrm{mg}$. hydrocortisone brings the nor-adrenaline excretion per $24 \mathrm{hrs}$ from 1.5 gammas to $23 \cdot 71$ gammas, and the excretion ratio from $1 \cdot 2$ to 14 , which shows that the secretion potential or nor-adrenaline is unchanged.

(c) The perfusion goes on for $8 \mathrm{hrs}$ with a one-litre solution of $0 \cdot 1$ per cent. procaine, a chemical substance that inactivates "L. amino-oxydase", destroying the ferment of nor-adrenaline, and brings the nor-adrenalinaemia in $8 \mathrm{hrs}$ from 1.08 gammas to 9.61 gammas and the excretion ratio from $0 \cdot 5$ to $7 \cdot 6$, which suggests that in rheumatoid arthritis there is an exaggerated destruction of nor-adrenaline by "L. aminooxydase".

Thus there seems to exist in rheumatoid arthritis an exaggerated destruction by " $L$. amino-oxydase" of the chemical transmitter of the sympathetic nervous system, nor-adrenaline, which phenomenon is expressed by a reversal of the ratio of urinary excretion Noradrenaline : Adrenaline Hydrocortisone at a sufficient dose, through a still unknown mechanism normalizes this ratio.

(10) Role of the Adrenals in Rheumatoid Arthritis. By Gunnar Birke and Lars-Olof Plantin (Stockholm, Sweden).
By a detailed investigation with several different methods, the role of the adrenals was investigated in rheumatoid arthritis, with the following results:

The total excretion of 17-ketosteroids in one hundred cases was within the lower limits of normal. The main 17-ketosteroids showed no qualitative deviation from normal and no unusual 17-ketosteroids could be identified. The 17-ketosteroid pattern changed as in normal subjects after ACTH and cortisone. The content of free hydrocortisone-like steroids (Porter-Silber chromogens) in the blood was normal in ten patients, as well as the increase after intramuscular ACTHstimulation; the excretion of corticosteroids (17-ketogenic steroids) was about 50 per cent. lower than in healthy persons. Twenty patients with rheumatoid arthritis, stimulated with 25 I.U. ACTH intravenously, showed a fall in the excretion of corticosteroids (17-OHCS by the modified method of Appleby and others) which was statistically significant.

As the series comprised both early and late manifestations of rheumatoid arthritis, further investigations will be made with patients in the early stages of the disease to see if this is a primary or secondary phenomenon.

$17-\alpha$-hydroxypregnanolone is excreted in the same amounts in rheumatoid arthritis as in healthy persons. The corticosteroid pattern, studied by a new method, was altered in four rheumatoid arthritis patients as compared with normal subjects. There was an increase in the ketonic corticosteroids (both 11-desoxy- and 11-oxysteroids); this altered steroid excretion was confirmed by another method of studying the problem.

Finally the cortico- and 17-ketosteroid excretion in pregnancy and hepatitis supports the hypothesis that the influence on rheumatoid arthritis depends on an altered steroid metabolism rather than on an increased secretion of adrenocortical steroids.

(11) Characterization of Rheumatoid and L.E. Factors. By Joseph Lospalluto, Georges Fallet, and Morris ZIFF (New York, U.S.A.).

Serum and euglobulin of patients with rheumatoid arthritis and systemic lupus erythematosus have been fractionated on anion and cation cellulose exchangers.

The rheumatoid factor was eluted from the anion exchanger in a fraction far removed from the main bulk of the gamma globulin. The active material, however, moved with the mobility of a slow moving component of the gamma globulin fraction when subjected to boundary electrophoresis. When the fraction containing the rheumatoid factor, derived from the anion exchanger, was chromatographed on a cation exchanger, two gamma globulin components were obtained, one of which demonstrated mainly sensitized sheep cell agglutinating activity, while the second precipitated with Fraction II and showed no agglutinating activity.

For measurement of L.E. factor activity a modification of the method of Schultz, Baum, and Ziff was employed, in which disks of living polymorphoneucleocytes are incubated on a glass slide with L.E. serum and serum fractions dissolved in normal serum. The L.E. factor 
was found in two gamma globulin peaks. When the eluates from each of these were concentrated and submitted to zone electrophoresis on polyvinyl chloride, the L.E. factor activity was found in each instance in a slowly moving component of the gamma globulin fraction. Ultracentrifugation of an active fraction derived from the cation exchanger demonstrated only protein with a sedimentation coefficient of 7 .

(12) Rheumatoid Haemagglutination Factor and its Significance in Differential Diagnosis. By N. SvarTZ (Stockholm, Sweden).

Investigations have been carried out (in collaboration with K. Schlossmann and L. Carlson) into the rheumatoid factor, that is to say, that factor in the blood of cases of rheumatoid arthritis which induces agglutination of sensitized erythrocytes.

These workers have demonstrated that the rheumatoid factor is precipitated in cold $\left(+4^{\circ} \mathrm{C}\right.$. $)$ after the serum has been diluted with distilled water. The haemagglutinating factor in other diseases is, as a rule, left in the supernatant fluid. The rheumatoid factor has been further purified by means of Zone-electrophoresis in a starch column. Further, attempts have been made to purify the factor through ultracentrifuging of cold precipitate from serum $(60,000$ r.p.m.). As a result of this centrifuging there were obtained a top fraction with a sedimentation constant of just over 6 Svedberg and a bottom fraction with a sedimentation constant of 19-20S. The top fraction produced no haemagglutination reaction, whereas the bottom fraction gave a strong haemagglutination reaction. Thus, the rheumatoid factor was found in a fraction that contained macroglobulins. Finally, attempts were made to isolate the rheumatoid factor by cation exchange (carboxymethylcellulose).

(13) Results of the Haemagglutination Test for Rheumatoid Arthritis with Cold-Precipitated Sera. By L. A. M. VAN Der SPeK and MARIA T. VervaAt (Amsterdam, Holland).

The results of the agglutination reaction with sensitized sheep blood in unmodified absorbed serum and cold-precipitated absorbed serum were compared in 249 sera. The conclusion was reached that the use of cold-precipitated serum increased the specificity of the test for rheumatoid arthritis.

A titre $>16$ in the agglutination test with coldprecipitated serum is practically an absolute proof of the presence of rheumatoid arthritis.

(14) Serological Tests in the Differential Diagnosis of Rheumatoid Arthritis and Ankylosing Spondylitis. By P. BARCeló (Barcelona, Spain).

A battery of serological tests, including the haemagglutination reaction and streptococcal agglutinins $L$ and $O$, have been used in differential diagnosis. It was found that these reactions were negative in most cases of ankylosing spondylitis, even if they showed the clinical pattern of rheumatoid arthritis.

In arthropathic psoriasis, the results were also negative or doubtful.
(15) Differential Sedimentation Test in Relation to the Problems of Rheumatoid Arthritis. By Harry COKE (London, England).

The principles of this test were described as a means of estimating a protein complex, which is readily dissociable by heat and which is increased above the normal value as a typical though non-specific feature of active rheumatoid disease. Its possible relation to macroglobulins and mucoproteins was suggested. The value of this estimation in diagnosis and in serial examinations was emphasized. Its use was demonstrated in assessing the optimal dosage in gold therapy. The quantitative estimation of this protein-complex was generally unaffected by steroids or by phenylbutazone. The development of the nomenclature of this protein complex as the tissue-reactivity-factor was explained. The specific reduction of this factor by gold, bismuth, and thorium-Xemanation, and its anabolic stimulation by BAL, cysteine, and the transaminase coenzyme pyridoxine was demonstrated.

(16) Blocking of Chymotrypsin Enzyme by Alphaglyco-proteins. By HARRY COKE (London, England).

The inherent rise in $\alpha_{1}$ and $\alpha_{2}$ globulin-carbohydrate complexes in active rheumatoid disease was correlated with the splenin substances of Ungar, the tryptic inhibitor of Coke, the chymotryptic inhibitor of Bodman, and the plasmin inhibitor of Page-Thomas. Curtain electrophoresis enabled the isolation of these inhibitors to specific protein fractions: the trypsin inhibitor in the $\alpha_{1}$ and $\alpha_{2}$ globulin fraction, and the chymotrypsin inhibitor between the $\alpha_{1}$ globulin and albumin groups. Chymotrypsin splits the $\alpha_{1}$-glycoprotein, releasing sialic acid. The two inhibitors are therefore separate entities. A second paper electrophoresis from the curtain tubes suggests that the chymotrypsin inhibitor is not a true $\alpha_{1}$-globulin complex; it does not stain on paper with the periodic acid-Schiff method.

(17) Laboratory Findings in the Differential Diagnosis of Rheumatoid Arthritis. By ERIC Jonsson (Stockholm, Sweden).

In rheumatoid arthritis (and other "collagen diseases"), plasma cholinesterase activity is decreased, the blood amino acid content is decreased, mucoprotein values are normal or elevated, sternal marrow changes are observed, and C-reactive protein is present in the joint exudate.

Modern clinical research has supplied us with many useful diagnostic tools, and the diagnosis of rheumatoid arthritis is not difficult in typical cases, but we still lack a means of diagnosing this disease in its earliest stages. In such early cases we can only watch the patient until typical symptoms appear.

(18) Electrophoretic Blood Protein Analysis in Rheumatoid Arthritis and Other Collagen Diseases. By B. OLHAGEN (Stockholm, Sweden).

Paper electrophoresis has a greater methodical error than the Tiselius method and therefore is less reliable. It also yields less information, because minor changes in the protein-bound carbohydrates and the lipoproteins

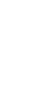


do not appreciably alter the pattern in the paper method. In the afebrile stages of rheumatoid disease one must rely upon other activity tests, such as polysaccharide analysis and C-reactive protein.

It would thus seem that the greatest merit of paper electrophoresis lies in the determination of gamma globulin. It gives no indication of the activity of the disease, but the extent of the systemic involvement of the mesenchymal tissue seems to be reflected in the gamma globulin content (except in intensively gold-treated patients). High gamma globulin values indicate systemic lupus erythematosus or visceral involvement in rheumatoid arthritis, as in Felty's syndrome and Sjögren's disease. Conversely, a normal gamma globulin in a patient with a high erythrocyte sedimentation rate and large articular effusions argues against rheumatoid arthritis and favours an acute disorder such as rheumatic fever and/or some other form of post-infectious polyarthritis.

(19) A Study of a Series of Rheumatoid Arthritic Patients treated with ACTH and biochemically Controlled. By W. S. C. Copeman (London, England).

A series of 76 severe cases of rheumatoid arthritis has been treated with self-administered injections of corticotropin over a period of 3 years. Sixteen of these patients have gone into clinical remission and have not relapsed. In a similar series of fifty patients treated with oral cortisone, only two showed clinical remission.

With the help of weekly biochemical estimations of the urinary 17-hydroxycorticosteroids by Norymberski's method, which gives a better measure of adrenocortical activity than any previous procedure has done, we were able to follow the level of adrenal stimulation produced, and so maintain optimal dosage throughout.

This appears to be a step forward in the scientific control of long-term steroid hormone therapy in rheumatoid arthritis.

A subsidiary finding was that the composition of commercially packaged ACTH may vary enormously from batch to batch. Until standardization can be assured, many of the results of treatment or of experimental work using corticotrophin must be considered unreliable.

(20) Long-Term Treatment of Rheumatoid Arthritis with Adrenocortical Steroids. By K. BRøCHNER-MORTENSEN and J. Boye Nielsen (Copenhagen, Denmark).

Fifty patients with rheumatoid arthritis were followed closely during treatment with adrenocortical steroids for up to 7 years. The treatment was discontinued in eight patients in whom the response was unsatisfactory, in eight with undesirable side-effects and complications, and in seven in whom the disease went into remission. Among 26 patients still under treatment with prednisone, the number of those able to work rose from one to twenty, and the number of those totally incapacitated fell from seven to one.

In the course of metabolic studies it was observed that prednisone caused only transitory negativity in the protein and sodium balance.
(21) Aspirin, Phenylbutazone, and Hormones in the Treatment of Rheumatoid Arthritis. By G. D. KersLey (Bath, England).

In the medicinal treatment of rheumatoid arthritis, aspirin compounds were the first choice. The relative advantages and disadvantages of different preparations were discussed, especially in relation to gastric irritation and bleeding. A method of desensitization in cases of true allergy by the use of steroids was outlined. Phenylbutazone was worthy of trial, if need be, in the co-operative or carefully supervised patient, but should be discarded within a week if not really effective. ACTH was found to be better than steroids for short-term treatment, but the difficulty of potency, sensitivity, and infection must be considered. Delta-steroids are better than cortisone in most cases if used in a ratio of $1: 5$, the greater number of gastric complications encountered was thought to be due to using them in too high doses.

(22) Serochemical Tests in Patients with Rheumatoid Arthritis during Treatment with Phenylbutazone and Prednisone. By R. Schoen (Göttingen, Germany).

To study and to compare the effects of phenylbutazone and prednisone in patients suffering from rheumatoid arthritis, a number of serochemical tests were performed and the data correlated with the clinical course of the disease. This investigation was conducted over a long period of time with a large number of patients. In a homogeneous series of forty moderately severe cases treated for 4 to 6 weeks, a comparison of the effects of thee two drugs gave the following results:

(1) The raised erythrocyte sedimentation rate was not influenced by phenylbutazone $(3(2) \times 0 \cdot 2 \mathrm{~g}$.), but was regularly improved by prednisone $(2 \times 10 \mathrm{mg}$.). In two-thirds of the cases the erythrocyte sedimentation rate returned to the normal range.

(2) Plasma fibrinogen levels initially exceeding $400 \mathrm{mg}$. in more than half of the patients were not lowered by phenylbutazone, but were usually brought to normal by prednisone.

(3) Electrophoresis showed that the serum albumin level (which in four-fifths of the cases was considerably diminished before treatment), returned to normal upon administration of prednisone in half the cases, and in the other half was raised above 40 per cent. The alpha globulin level remained unchanged. The gamma globulin level, which was much above normal in 90 per cent. of the patients, showed a striking fall. By contrast no significant effect was observed with phenylbutazone.

(4) On the whole the total serum proteins were raised by prednisone and lowered by phenylbutazone.

(5) Hypopotassaemia was seen in 55 per cent. of the patients treated with prednisone and in 15 per cent. of those given phenylbutazone. The sodium level remained unchanged.

(6) The anti-streptolysin titre and the concentration of serum mucoproteins (which fluctuated considerably) showed no significant reaction.

(7) The hyaluronidase-inhibition test showed no change during phenylbutazone administration, but gave normal values during prednisone therapy. 
(8) Data on the concentration of C-reactive protein and on the diphenylamine reaction were inconclusive.

(23) Various Combinations of Therapy in the Treatment of Rheumatoid Arthritis. By A. ROBECCHI (Turin, Italy).

Since there is no specific treatment for rheumatoid arthritis, it is advisable to attack the disease with several drugs simultaneously, in order to slow down the inflammation and avoid the development of irreversible lesions. Salicylates, pyramidon, phenylbutazone, gold salts, and various adrenocortical steroids have been given in combination; the most interesting results were obtained by the administration of gold and prednisone in a series of 48 patients. Salicylates by mouth and hydrocortisone and prednisolone by intra-articular injection may be given at the same time in particularly severe cases.

(24) Side-Effects after 5 Years' Continuous Use of Corticosteroids. By M. VirKKunen and L. Lehtinen (Helsingfors, Finland).

Information on how far prolonged corticosteroid treatment adds to the hazards of these hormones and how far the side-effects contraindicate continued therapy is still inadequate. To elucidate these questions, a series of 19 cases treated with corticosteroids for five years or more was studied. Observations were also made on the occurrence of peptic ulcer and tuberculosis during steroid medication. The incidence of side-effects increases, though not alarmingly, as treatment is prolonged. Two cases of osteoporotic fractures of the spine improved during continued treatment. In nine cases of peptic ulcer clinical and roentogenological healing was satisfactory during corticosteroid therapy, with only one recurrence during a follow-up of 2 to 30 months. This suggests that peptic ulcer is often only a single episode during steroid therapy. Tuberculosis developed in five cases as a flare-up of primary dissemination. Since the signs of healed primary tuberculosis are negligible or impossible to detect, dangers attending steroid therapy in a population highly infected with tuberculosis are considerable.

(25) Inflammatory Rheumatism in Old People. By F. Françon (Aix-les-Bains, France).

Only cases of rheumatism which begin after the age of 50 are considered below, conditions resulting from previous rheumatic affections being ignored. Apart from the presence of rheumatic disease, old age is always accompanied by a raised erythrocyte sedimentation rate, and the development of osteoporosis (especially in women).

Rheumatic Fever.-This is rare after the age of 25 , but may occur in old people. It begins with a sore throat, which is followed after an interval of about 10 days by a sharp rise in the antistreptolysin-O titre. The arthritis is less acute, the carditis less severe, and the prognosis better than in children, but the attack is often followed by chronic articular lesions. Treatment with penicillin and prednisone is recommended.
Rheumatoid Arthritis.-30 per cent. of cases begin after the age of 50, and male cases are more amongst the older patients.

In the fifties the disease runs its course in three phases:

(1) Mode of Onset.-This may be acute as in cases of gout or rheumatic fever, insidious as in typical rheumatoid arthritis, asthenia and tiredness preceding the polyarthritis, or may be secondary to a previous osteoarthritis.

The joints first involved are the metacarpo-phalangeal or proximal phalangeal of the second and third fingers. The arthritis is accompanied by swelling, amyotrophy, contracture, stiffness, ankylosis, deformity, and severe constitutional disturbances.

(2) Period of Progress.-The arthritis evolves in a series of acute attacks and the invasion of new joints culminate in a pseudo-symmetry. The deformity is irreversible with marked trophic disorders.

(3) Period of Stabilization.-The pain disappears and the serological reactions gradually return to normal. The patient may be crippled but his systemic condition is usually fairly good.

In the sixties there is no outstanding aetiological feature and the onset is generally insidious. The shoulders and hips are often involved, there is no sharp pain, nor ulnar deviation. The general condition is poor but recurrences are rare.

In the seventies we find acropolyarthritis attacking mainly the upper limbs. Slight deformities and cyanosed extremities are accompanied by a good general condition, a high erythrocyte sedimentation rate, and pronounced osteoporosis.

As a rule the prognosis is good and therapy effective.

Gold therapy is well tolerated and effective, as is intraarticular hydrocortisone acetate. Calcium, vitamins C and D, oestrogens, and androgens may also be tried. Prednisone is still under trial.

Ankylosing Spondylitis.-Two types are seen:

(1) Slow progress and late appearance.-Clinically it only becomes obvious in old age, but lesions may be detected much earlier by $x$ ray.

(2) Beginning in the fifties with incomplete sacro-iliac lesions and syndesmophytes covering osteophytes. The differential diagnosis should exclude chronic vertebral hyperostosis. Gold therapy is recommended.

Rhizomelic Pseudo-Polyarthritis.-This usually begins about the age of 50 and is commoner amongst females. After an acute phase, it progresses more slowly with involvement of both shoulders. No $x$-ray alterations are seen. The prognosis is favourable.

After 50 years of age, the chronic inflammatory rheumatisms evolve slowly; their progress is limited and patients show a good response to treatment.

(26) Salicylate Activity. By H. van Cauwenberge and J. Roskam (Liége, Belgium).

In 1951, several workers were led by their clinical and experimental investigations to conclude that salicylates 
induce an hypothalami-hypophyso-adrenocortical hyperactivity.

In animals, especially in the rat, ACTH and sodium salicylate administered at sufficiently high doses induce the same haematological, biochemical, and histological modifications. These two drugs increase the level of the free and total 17-hydroxycorticosteroids in the serum as well as reducing the steroids in the plasma.

On the other hand, in man, the salicylates induce modifications similar to those occurring in a Cushing's syndrome, these changes being due to adrenocortical hyperactivity. They also cause a fall in the number of circulating eosinophils after the intake of $6 \mathrm{~g}$., and (in patients who respond to the treatment) an increase in the free and total serum 17-hydroxycorticosteroids and the urinary 17-hydroxy- and reducing steroids.

This suggests that salicylates stimulate the hypophysoadreno-cortical axis. These drugs may have other specific pharmacodynamic activities on the peripheral tissues, which we have investigated in various experiments.
Psychogenic Rheumatism, its Incidence, Practical Importance, and Differentiation from Rheumatoid Arthritis and Fibrositis. By P. S. Hench (Rochester, Minn., U.S.A.).

Differential Diagnosis between Rheumatoid Arthritis and Ankylosing Spondylitis. By J. Forestier (Aix-lesBains, France).

Chronic Inflammatory Rheumatism following Rheumatic Fever. By J. Graber-Duvernay (Aix-les-Bains, France).

Surgical Intervention in Arthritis. By S. FrIBERG (Stockholm, Sweden).

Osteoporosis and Fractures complicating Steroid Therapy for Rheumatoid Arthritis. By EDWARD F. Rosenberg (Chicago, Ill., U.S.A.).

\section{BOOK REVIEW}

Patologia rara delle articolazioni. By Claudio Cervini and Cesare Longo. 1957. Pp. 297, 28 figs. Edizioni Medichi e Scientifichi, Rome. (L. 3,500; \$6; 42s.)

This volume is a product of the Rome School of Rheumatology. The authors have included as many rare affections of joints as possible but, as a definition of rarity under the circumstances would tax even the resources of a Parkes-Weber, it is not surprising to find, for instance, that over forty pages have been devoted to psoriatic arthritis. Print and paper are of excellent quality and the task of the reader has been eased by the provision of a table of contents, a list of authors, and an extensive international bibliography at the end of each chapter. Very thoughtfully, too, the publishers have supplied a plastic dust-cover which is both transparent and substantial-an innovation which should be more generally copied.

This volume can only have a limited appeal, though the references provided open interesting vistas for research workers. DAvid Preiskel. 